\title{
Robotic Eye Surgery: Past, Present, and Future
}

\section{John D. Pitcher ${ }^{1}$, Jason T. Wilson ${ }^{2}$, Tsu-ChinTsao ${ }^{2}$, Steven D. Schwartz ${ }^{1}$ and Jean-Pierre Hubschman ${ }^{1 *}$}

${ }^{1}$ Jules Stein Eye Institute, Department of Ophthalmology, University of California, David Geffen School of Medicine, Los Angeles, USA ${ }^{2}$ Department of Mechanical and Aerospace Engineering, University of California, Los Angeles, USA

\begin{abstract}
Purpose: To review past attempts, current innovations, and future goals of robotic eye surgery.

Methods: A Medline literature search using the words "robot" and "ophthalmology" was performed to identify all relevant literature. Pertinent articles were reviewed and content summarized based on context.

Results: Purported potential benefits of robotic-assisted eye surgery include improved precision, reduced tremor, amplified scale of motion, and the potential of automation and telesurgical operation. Several investigators have created devices capable of performing individual intraocular tasks, and efforts are underway to develop platforms designed to allow completion of entire ophthalmic procedures.
\end{abstract}

Conclusion: Although obstacles such as cost and availability exist, prior successes and future benefits of robotic eye surgery are promising reasons for the continuation of research efforts.

Keywords: Robot; Da vinci; Cataract surgery; Ophthalmology

\section{Introduction}

Robotic surgical systems have had an increasing presence in the surgical landscape over the past two decades. Although the most wellknown platform is the Da Vinci Surgical System (Intuitive Surgical, Sunnyvale, CA), there are hundreds of different devices in use for abdominal, urologic, pelvic, cardiovascular, and neurologic surgery [1]. Robot-assisted methods offer many advantages over conventional surgical techniques such as improved precision, reduced tremor, and amplified scale of motion (Table 1). It is no surprise that we are in the midst of a paradigm shift towards the integration of robotic augmentation of conventional surgical methods.

Ophthalmic surgeries are commonly performed with a highmagnification, three-dimensional view through a surgical microscope. Precise manipulations must be performed using delicate handheld instruments to minimize collateral damage that might result in a poor visual outcome for the patient (Figure 1a). Robotic surgery is particularly attractive in these cases, because of the advantages listed above. Complex feedback loops (Figure 1b) present numerous challenges to investigators who have attempted the adaptation or development of robotic devices to accomplish intraocular surgical maneuvers. Here we review prior advancements, current innovations, and future directions of robotic eye surgery.

\section{Previous Advancements in Robot-Assisted Ophthalmic Surgery}

Historically, most innovations in the field have focused on either

\begin{tabular}{|l|l|}
\multicolumn{1}{|c|}{ Advantages } & \multicolumn{1}{c|}{ Disadvantages } \\
\hline Precision, accuracy, stability & Poor decision making/judgement \\
\hline Amplified scale of motion & Poor interpretation of qualitative data \\
\hline Reduced tremor & Expense and maintenance \\
\hline Multitasking & Availability \\
\hline Automation & Learning curve \\
\hline Association of imaging systems & Possibility of malfunction \\
\hline Teleoperation & Patient trust \\
\hline
\end{tabular}

Table 1: Advantages and disadvantages of robotic surgery. accomplishing single tasks or assisting in technically difficult portions of procedures. One of the earliest examples was a micro-manipulator developed by investigators in France in the late 1980's. This device was one of the first to attempt maintenance of a remote center of motion (RCM), a fundamental necessity in intraocular surgery to avoid tissue damage due to translational forces at points of entry. The Stereotaxical Micro-telemanipulator for Ocular Surgery (S.M.O.S) allowed for 4 degrees of freedom (DoF) including rotation and translation about the RCM. A carrier allowed for 3-dimensional movement during surgery. Precise movements could be completed at the expense of greatly increased surgical procedure time [2]. Over the next decade, several other groups described prototypes with the ability to perform dedicated microsurgical tasks in animal models, including cannulation of retinal vessels [3], drainage device implantation [4] intravascular drug delivery, and microvascular pressure measurement [5].

Japanese collaborators created a prototype robotic system based on the S.M.O.S. platform that is designed to aid in multiple steps of vitreoretinal surgery. Robotic assistance increased accuracy five to ten fold, independent of the surgeon. The system eliminated the interoperator variability in precision that is seen in similar tasks performed manually. These modifications facilitated successful surgical induction of a posterior vitreous detachment, retinal vessel sheathotomy using 25 -gauge microscissors, and microcannulation of retinal vessels with a diameter of 100 microns in porcine eyes [6].

Investigators at Johns Hopkins University developed a steadyhand manipulator (SHM) for retinal microsurgery [7]. This device consists of an arm with tilt and roll mechanisms on an xyz-stage that is

*Corresponding author: Jean-Pierre Hubschman, Jules Stein Eye Institute, 100 Stein Plaza, Los Angeles, CA 90095-7000, USA, Tel: (310) 206-5004; Fax: (310) 794-7905; E-mail: hubschman@jsei.ucla.edu

Received February 04, 2012; Accepted May 16, 2012; Published May 19, 2012

Citation: Pitcher JD, Wilson JT, Tsao TC, Schwartz SD, Hubschman JP (2012) Robotic Eye Surgery: Past, Present, and Future. J Comput Sci Syst Biol S3:001. doi:10.4172/jcsb.S3-001

Copyright: (c) 2012 Pitcher JD, et al. This is an open-access article distributed under the terms of the Creative Commons Attribution License,which permits unrestricted use, distribution, and reproduction in any medium, provided the original author and source are credited. 
attached to a force/torque sensor [6] allowing the instrument to move at the direction of the surgeon with software-augmented proportional velocity. The design places the RCM at the sclera, minimizing undesirable tension on the eye wall. The SHM provides filtration of tremor that was demonstrated experimentally. The operators, with an average of 182 microns of tremor, were successful in cannulating80 micronchorioallanotoic veins in chicken embryos. Further innovations by this group include intra-operative retina registration that syncs with pre-operative imaging to guide treatment delivery [8]. Equally as impressive is the Micron, a microsurgical tool that reduces unintentional tremor while preserving eye-hand coordination. Surgeons experienced up to $52 \%$ reduction in error in three simple microscopic positioning tests [9].

For even more dexterous manipulations, a group from Columbia University recently proposed a theoretical multi-arm hybrid robotic system [10] and a 16-DoF system utilizing surgical tools inside of the eye itself [11]. Other investigators have also proposed intraocular robots as a minimally invasive mechanism to provide a high degree of mobility and precision in the placement of intravitreal inserts for treatment of exudative age related macular degeneration. Using micro robots with an outer diameter of less than $500 \mu \mathrm{m}$, the authors were able to achieve targeted placement of drug reservoirs in porcine eyes using wireless electromagnetic controls [12].

\section{The Present Dilemma: Complete Procedures}

In the past, ophthalmic robotic surgical systems have focused on single tasks, often those that are particularly delicate. Although much of this research is ongoing, interest is growing in designing devices that are able to perform the entire surgical procedure, with the goal of adding speed and efficiency without sacrificing precision. Requirements for this goal include sufficient range of motion, simultaneous surgical instrument manipulation, and capability of mid-surgery instrument switching.

The Da Vinci Surgical System, approved by the Food and Drug Administration in 2000, increased the number of general robotic surgical procedures by a factor of $15 \mathrm{in}$ its first four years of use [13]. The system consists of two components: the mechanical robot apparatus with three or four arms with a dual channel endoscope (Figure 2a) and a control console where the surgeon manipulates the robotic arms remotely while looking through a stereoscopic viewfinder (Figure 2b). Each robotic arm can tilt in two planes and pivot around a stable point of rotation.

In ophthalmic surgery, this system has been used to perform suture repair of a corneal laceration [14], complete a continuous capsulorhexis on the anterior lens capsule in cataract surgery, and perform a 3-port 25-gauge pars plan a vitrectomy (Figure 2c) in porcine eyes [15]. Several limitations of the Da Vinci Surgical System were noted. The robotic arms did not mirror the exact movements of human arms, preventing a perfectly round, curvilinear capsulorhexis that would be optimal for cataract surgery. In addition, the $5 \mathrm{~cm}$ distance between the RCM and the instrument tip (site of ocular penetration) limited motion and created undue tension on the external eye surface. The endoscope lacks retroillumination capabilities and its position inhibited peripheral vitreous gel removal.

To overcome these challenges, investigators have customized microsurgical systems for ophthalmic surgeries. By mounting a micro
A)

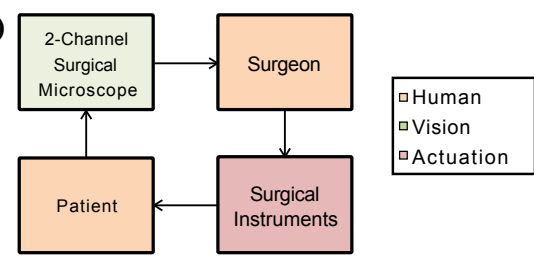

B)

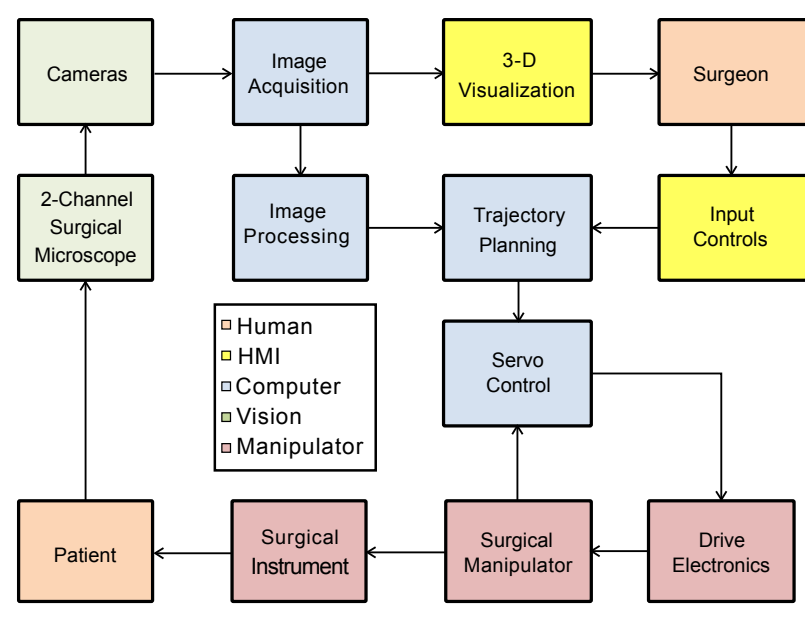

Figure 1: Feedback loop during standard ophthalmic surgery. b. Feedback loop during robotic-assisted ophthalmic surgery.
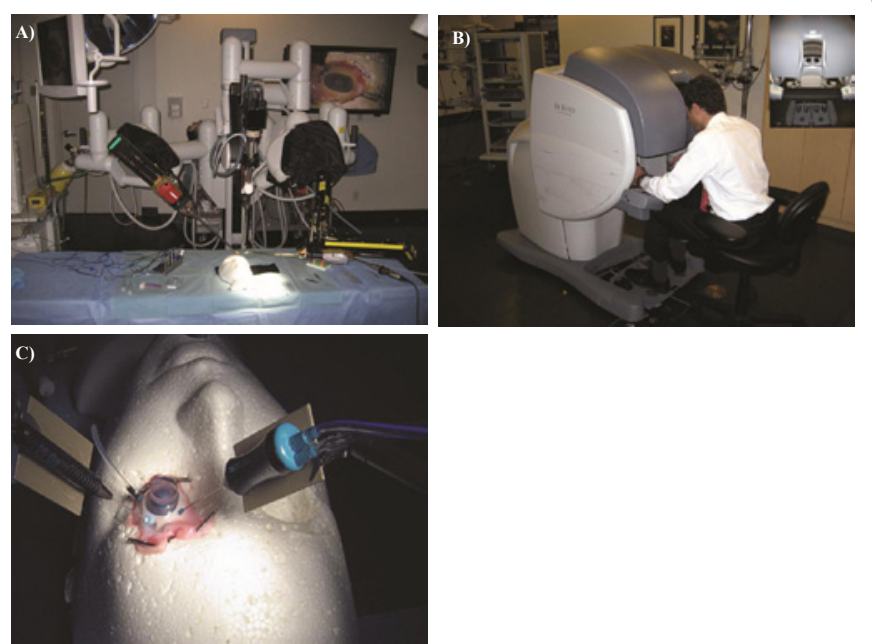

Figure 2: The da Vinci Surgical System for ophthalmic microsurgery [15] a. One central arm holds the endoscope; two side arms (green and yellow stripes) hold surgical instruments. b. The operator obtains a three-dimensional view of the surgical field and accesses the controls at the console.c. Insertion of the modified 25-gauge vitreous cutter and endoilluminator with the robotic arms.

robot, the Hexapod Surgical System (HSS), to the Da Vinci macro robot, a remote center of motion at the site of ocular penetration can be achieved (Figure 3). The precision and dexterity of this approach was validated by successful insertion of a vitreous cutter through a sclerotomy in porcine eyes [16]. Another adaptation, known as the "Micro hand," was equipped with micro electromechanical systems (MEMS) technology. This device was designed to mimic a human hand and is pneumatically controlled, allowing titration of grasping force (Figure $4 \mathrm{a}$ ). Four fingers, with a length of $4 \mathrm{~mm}$ each (Figure $4 \mathrm{~b}$ ), were 
Citation: Pitcher JD, Wilson JT, Tsao TC, Schwartz SD, Hubschman JP (2012) Robotic Eye Surgery: Past, Present, and Future. J Comput Sci Syst Biol S3:001. doi:10.4172/jcsb.S3-001

used to maneuver caliper weights and manipulate fresh retinal tissue of porcine cadaver eyes at $60 \mathrm{psi}$ of applied compressed air [17].

The Intraocular Robotic Interventional Surgical System (IRISS), a joint effort between the Jules Stein Eye Institute and the UCLA Department of Mechanical and Aerospace Engineering, is a dedicated microsurgical platform capable of performing complete ophthalmic procedures. The master-slave design features a remote console, similar to the Da Vinci and Eye RHAS (Eye Robot for Haptically Assisted Surgery) systems [18], which could facilitate telesurgery. The IRISS design includes a head-mounted "True Vision" (True Vision Displays, Inc., Cerritos, CA) stereoscopic visualization system, two joystick controls with tremor filtration and scaled motion, custom designed

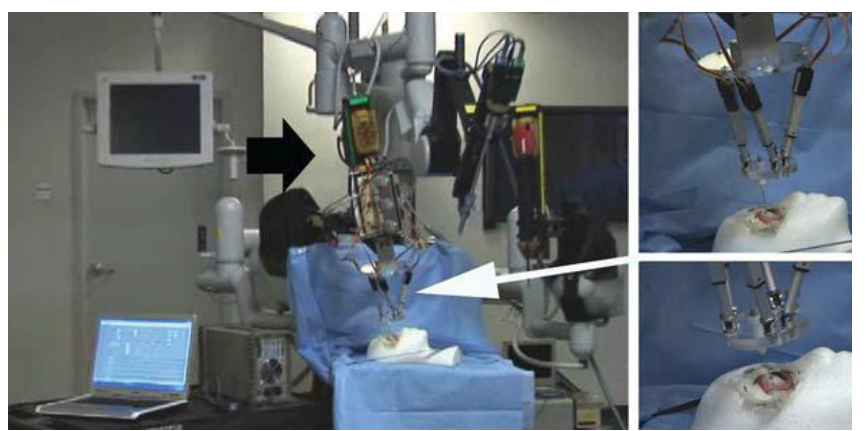

Figure 3: The Hexapod Surgical System (HSS) integrates with the da Vinci platform (black arrow) to create a micro-macro robotic system. Six linear actuators change length when remotely commanded, allowing control of a vitrectomy probe held by the HSS arms (white arrow) [16].
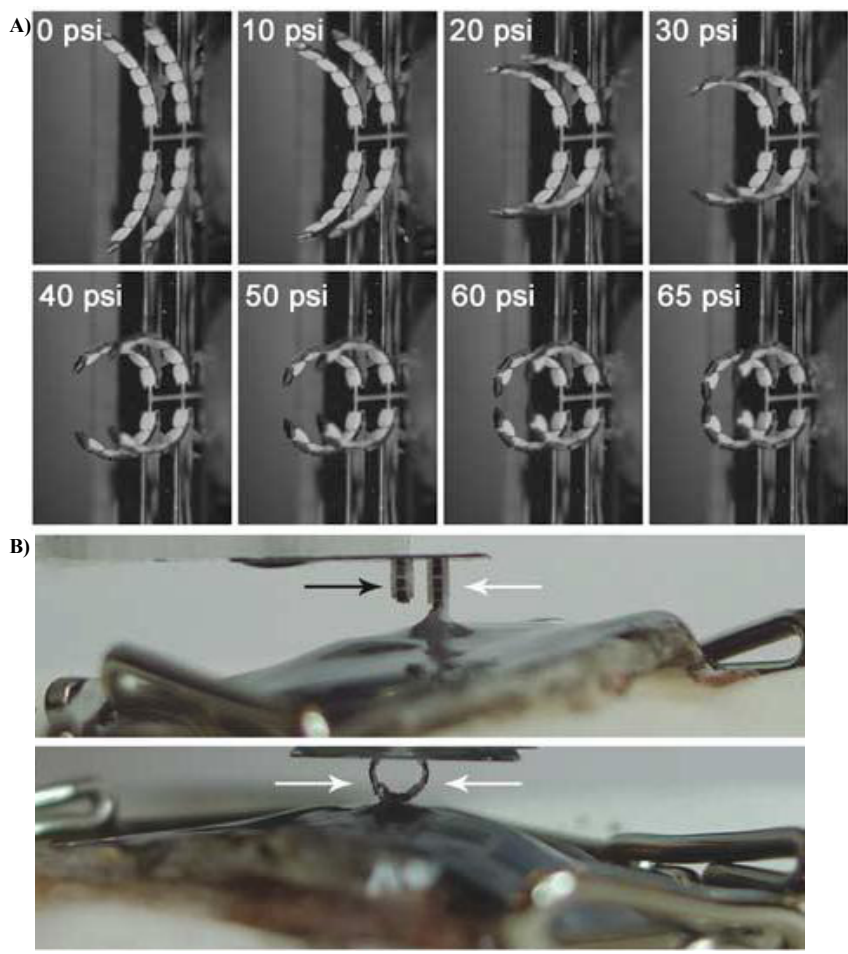

Figure 4: The "microhand" for ophthalmic microsurgery [17]. a. Each micro finger progressively closes under increasing air pressure. At above $65 \mathrm{psi}$, a grasping force is created. b. Two opposing fingers (black stripes) are able to lift retina when closed pneumatically (white arrows).
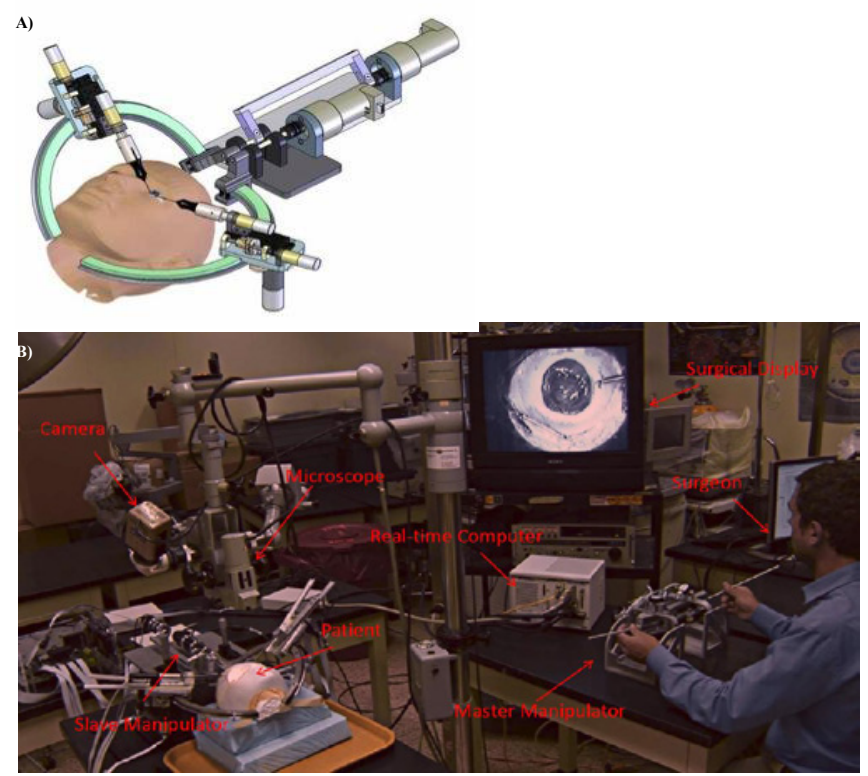

Figure 5: The Intraocular Robotic Interventional Surgical System (IRISS). a. Animated isometric view. b. Experimental setup.

arms appropriately sized to accommodate commercially available instrumentation, and two closely approximated remote centers of motion to avoid stress on surrounding tissues (Figure 5). In ongoing early trials, validation in porcine eyes has focused on three complex ocular procedures: lens capsulorhexis in cataract surgery, 23-gauge core vitrectomy, and retinal vein microcannulation.

\section{The Future of Robot-assisted Eye Surgery: Automation and Integration}

In the past several years, advances in ophthalmologic imaging modalities such as optical coherence tomography (OCT) and ultrasound biomicroscopy (UBM) have enhanced the surgeon's ability to localize pathology both pre- and intra-operatively [19]. Simultaneously, femtosecond laser devices have been optimized for wound construction, capsulorhexis creation, and nucleus breakdown during cataract surgery. Integration of a robotic system with OCT has been proposed for vitreoretinal surgery [20], and the addition of laser technology would facilitate automation of surgery to treat cataract, which is a leading cause of blindness worldwide. Laser refractive surgery, such as laser-assisted in situ keratomileusis (LASIK), has already become nearly completely automated and requires minimal intra-operative manipulation by the surgeon. For less standardized procedures, such as vitreoretinal surgery, robotic augmentation could ultimately increase efficiency, amplify scale to allow performance of otherwise difficult tasks (e.g. sub retinal delivery of medication or stem cells), decrease complication rates by reducing tremor and increasing precision, and permit telesurgical care in remote locations.

Several obstacles remain before robotic surgery will become clinical reality in ophthalmology. High cost, steep learning curve, and patient trust all present individual challenges. These hurdles are similar to those initially encountered by the proponents of minimally invasive laparascopic surgery, which is now a widely accepted technique [21]. Over two decades of evidence suggests that robotic devices may help facilitate higher quality of care and that research in the field should be continued. 
Citation: Pitcher JD, Wilson JT, Tsao TC, Schwartz SD, Hubschman JP (2012) Robotic Eye Surgery: Past, Present, and Future. J Comput Sci Syst Biol S3:001. doi:10.4172/jcsb.S3-001

\section{References}

1. Thiel DD, Winfield HN (2008) Robotics in urology: past, present, and future. $J$ Endourol 22: 825-830.

2. Guerrouad A, Vidal P (1989) SMOS: Stereotaxical micro-telemanipulator for ocular surgery. Engineering in Medicine and Biology Society: Proceedings of the Annual International Conference of the IEEE Engineering 3: 879-880.

3. Grace KW, Colgate JE, Glucksberg, Chun JH (1989) A six degree of freedom micromanipulator for ophthalmic surgery. Engineering in medicine and biology society proceedings $3: 879-880$.

4. Yu DY, Cringle SJ, Constable IJ (1998) Robotic ocular ultramicrosurgery. Aust N Z J Ophthalmol 26 Suppl 1:S6-8.

5. Jensen PS, Grace KW, Attariwala R, Colgate JE, Glucksberg MR (1997) Toward robot-assisted vascular microsurgery in the retina. Graefes Arch Clin Exp Ophthalmol 235: 696-701.

6. Uneri A, Balicki MA, Handa J, Gehlbach P, Taylor RH, et al. (2010) New steady-hand eye robot with micro-force sensing for vitreoretinal surgery. Proc IEEE RAS EMBS Int Conf Biomed Robot Biomechatron.

7. Mitchell B, Koo J, lordachita I, Kazanzides P, Kapoor A, et al. (2007) Development and application of a new steady-hand manipulator for retinal surgery. IEEE ICRA 623-629.

8. Fleming IN, Voros S, Vagvolgyi B, Pezzementi Z, Handa J, et al. (2008) Intraoperative visualization of anatomical targets in retinal surgery. Applications of Computer Vision, IEEE Workshop 1-6.

9. MacLachlan RA, Becker BC, Tabarés JC, Podnar GW, Lobes LA, et al (2012) Micron: An actively stabilized handheld tool for microsurgery. IEEE Transactions on Robotics 28: 195-212.

10. Wei W, Goldman RE, Fine HF, Chang S (2009) Performance evaluation for multi-arm manipulation of hollow suspended organs. IEEE Transactions on Robotics 25: 147-157.

11. Wei W, Goldman RE, Simaan N, Fine H, Chang S (2007) Design and theoretical evaluation of micro-surgical manipulators for orbital manipulation and intraocular dexterity. Proc of the Int Conf on Robotics and Automation.

12. Bergeles C, Kummer MP, Kratochvil BE, Framme C, Nelson BJ (2011)
Steerable intravitreal inserts for drug delivery: In vitro and ex vivo mobility experiments. Proc of the 14th Int Conf on Medical Image Computing and Computer Assisted Intervention.

13. Kumar R, Hemal AK (2005) Emerging role of robotics in urology. J Min Access Surg 1: 202-210.

14. Tsirbas A, Mango C, Dutson E (2007) Robotic ocular surgery. Br J Ophthalmo 91: 18-21.

15. Bourla DH, Hubschman JP, Culjat M, Tsirbas A, Gupta A, et al. (2008) Feasibility study of intraocular robotic surgery with the da Vinci Surgical System. Retina 28: $154-158$

16. Bourges JL, Hubschman JP, Wilson J, Prince S, Tsao TC, et al. (2011) Assessment of a Hexapod Surgical System for robotic micro-macro manipulations in ocular surgery. Ophthalmic Res 46: 25-30.

17. Hubschman JP, Bourges JL, Choi W, Mozayan A, Tsirbas A, et al. 'The Microhand:' a new concept of micro-forceps for the ocular robotic surgery. Eye 24: 364-367.

18. Meenink HCM (2011) Vitreo-retinal eye surgery robot: sustainable precision

19. Robin R, Baranano DE, Fortun JA, Schwent BJ, Cribbs BE, et al. (2011) Intraoperative microscope-mounted spectral domain optical coherence tomography for evaluation of retinal anatomy during macular surgery. Ophthalmology 118: 2212-2217.

20. Balicki M, Han JH, lordachita I, Gehlbach P, Handa J, et al. (2009) Single fiber optical coherence tomography microsurgical instruments for computer and robot-assisted retinal surgery. Proc of the 12th Int Conf on Medical Image Computing and Computer Assisted Intervention.

21. Breitenstein S, Nocito A, Puhan M, Held U, Weber M, et al. (2008) Roboticassisted versus laparoscopic cholecystectomy: outcome and cost analyses of a case-matched control study. Ann Surg 247: 987-993

22. Herron DM, Marohn M (2008) A consensus document on robotic surgery. Surg Endosc 22: 313-325

23. Ueta T, Yamaguchi Y, Shirakawa Y, Nakano T, Ideta R, et al. (2009) Robotassisted vitreoretinal surgery: Development of a prototype and feasibility studies in an animal model. Ophthalmology 116: 1538-1543.

This article was originally published in a special issue, Assistive Portable Robots Design handled by Editor(s). Dr. Bozinovski Stevo, South Carolina State University, USA 